\title{
Optical residue arithmetic: a correlation approach
}

\author{
Demetri Psaltis and David Casasent
}

\begin{abstract}
A numerical optical processor is described that performs operations in residue arithmetic. The position coding used to represent decimal and residue numbers allows one to describe the various conversions and operations in a correlation formulation. This description of residue arithmetic leads directly to novel residue adder and decimal/residue/decimal converter designs, which are described and experimentally demonstrated. The accuracy, dynamic range, and space bandwidth of an optical residue arithmetic processor are also discussed.
\end{abstract}

\section{Introduction}

Residue arithmetic has been of interest because it is one of the fastest computing methods available. ${ }^{1-3}$ Huang ${ }^{4}$ was the first to suggest that the parallel arithmetic computational properties of residue arithmetic made the realization of such optical numerical processors attractive. Since then considerable advances ${ }^{5-7}$ have occurred in optical residue arithmetic, including a recent conference at Ohio State University. In this recent work, at least three distinguishable ways have been suggested for representing numbers in an optical residue system. ${ }^{7}$ These include phase or polarization coding and pulse-position coding. Collins ${ }^{6}$ has chosen the former method and Huang et al. ${ }^{7}$ the latter approach.

An extensive review of many of the possible technological and architectural realizations of optical residue arithmetic processors has recently been published. ${ }^{7,8}$ Many of the more advanced and promising approaches suggested for this new type of optical arithmetic system utilize integrated optical switches and planar waveguides, ${ }^{7}$ and the cyclic control and system operations are described by maps.

Residue arithmetic has not yet been described in the conventional terminology of signal processing and pattern recognition researchers. In this paper we attempt to close this gap by formulating optical residue arithmetic processors in linear systems, Fourier transform, and correlation terms. This approach allows one to realize more easily a residue arithmetic system in

\footnotetext{
The authors are with Carnegie-Mellon University, Department of Electrical Engineering, Pittsburgh, Pennsylvania 15213.

Received 14 June 1978.

0003-6935/79/020163-09\$00.50/0

(C) 1979 Optical Society of America.
}

terms of the many technologies that use correlators (CCD, SAW, $\mathrm{I}^{2} \mathrm{~L}$, integrated optics, etc.). We consider only coherent optical techniques in the specific system designs to be described, thereby utilizing the space bandwidth, parallel processing, and real time throughput of such systems. However, the correlation formulation presented is general enough to allow residue systems to be realized using other technologies. The specific optical system architectures presented and experimentally demonstrated are included to better convey the operations required and as examples of transitions from theory to system architecture to experimental verification.

A correlation-based formulation of residue arithmetic is most readily achieved if we use pulse-position coding as suggested by Huang et al. 5,7 to represent decimal and residue numbers rather than the phase or polarization of light. ${ }^{6}$ The decimal/residue/decimal converters we discuss can thus be directly interfaced to integrated optical $^{7}$ and other residue systems that also use pulseposition coding.

Although many optical processing researchers ${ }^{4-8}$ and others ${ }^{1-3}$ reviewed the advantages of residue arithmetic, a brief summary of its features follows, both for motivation and for completeness.

Residue arithmetic is especially attractive for optical implementation because it allows arithmetic operations to be performed in parallel since no carries occur. Also in residue arithmetic, a given computation is divided into subcomputations of reduced complexity. This allows these suboperations to be performed in parallel with reduced dynamic range requirements. Finally, the accuracy of the resultant operations is high because the system's dynamic range is proportional to the product of the residue moduli used. Thus, residue arithmetic directly allows the use of multichannel parallel computations that are the forte of optical computing without the dynamic range and accuracy limitations $\mathrm{s}^{9,10}$ normally associated with optical processors. 
In Sec. II, we briefly review residue arithmetic. In Secs. III and IV we describe decimal/residue/decimal converter designs to convey the pulse-position coding, carrier modulation, and aperture control operations needed in our suggested optical implementations of a residue arithmetic processor and for insight into the required processing. In Sec. $V$ we then present a general formulation of these conversion operations in correlation terminology rather than by use of polynomials and maps as provided in Ref. 7. An optical residue arithmetic adder is then described in Sec. VI in correlation terminology. In Secs. VII and VIII experimental demonstrations of coherent optical residue arithmetic are included, and the accuracy and space bandwidth features of such systems are discussed.

\section{Residue Arithmetic}

A brief review of residue arithmetic follows. It is included for completeness and to provide a brief introduction to this subject to those not familiar with it. This also allows us to define the notation to be used and to demonstrate clearly why the optical realization of a numerical processor using residue arithmetic is attractive.

An integer is represented in the residue number system by the $N$-tuple set of residues $\left(R_{m_{1}}, R_{m_{2}}, \ldots\right.$ $R_{m_{N}}$ ) with respect to the $N$ different relatively prime integer moduli $m_{1}, \ldots, m_{N}$. The residue $R_{m_{i}}$ is the least positive integer remainder of the quotient of $X$ and $m_{1}$. The maximum integer value that can be represented by $N$ moduli is $M-1$, where

$$
M=\prod_{i=1}^{N} m_{i}
$$

An example will easily clarify this. To represent 13 in the residue system with moduli $(5,7,9,2)$, we divide 13 by the first modulus 5 and obtain 2 and a remainder of 3. Dividing 13 by the second modulus 7, we obtain 1 and a remainder of 6 . Dividing 13 by $m_{3}=9$, the remainder is 4. For $m_{4}=2$, the remainder is 1 . These four remainders are the representation of 13 in the residue number system with moduli $(5,7,9,2)$, i.e., $13=$ $(3,6,4,1)$.

To see the advantages of residue arithmetic, we consider how to realize the sum of 13 and 59 in residue with moduli $(5,7,9,2)$. Proceeding as before, we find 59 $=(4,3,5,1)$. To sum two numbers in residue notation, we find $59=(4,3,5,1)$. To sum two numbers in residue notation, we sum each separate pair of residue numbers in the same modulus $m_{i}$ and retain only the residue, i.e.,

$$
\begin{aligned}
m_{i} & =(5,7,9,2) \\
\hline 13 & =(3,6,4,1) \\
+59 & =(4,3,5,1) \\
\hline 72 & =(2,2,0,0)
\end{aligned}
$$

where $3+4$ modulo 5 has a remainder $2,1+1$ modulo 2 is 0 , etc. Checking, we find that the decimal sum 72 is $(2,2,0,0)$ in moduli $(5,7,9,2)$.
To subtract two numbers in residue, e.g., 72-13, we invert 13 in residue (by simply complementing each bit $i$ modulo $m_{i}$ in the residue representation), i.e.,

$$
\begin{aligned}
m_{i} & =(5,7,9,2) \\
\hline 13 & =(3,6,4,1) \\
-13 & =(2,1,5,1) .
\end{aligned}
$$

The bits $i$ of the residue numbers 72 and -13 are then added as before:

$$
\begin{aligned}
72 & =(2,2,0,0) \\
-13 & =(2,1,5,1) \\
\hline 59 & =(4,3,5,1) .
\end{aligned}
$$

To multiply two numbers in residue, we convert each number to its residue representation, multiply each bit pair, and retain the residue of each product modulo $m_{i}$. Consider forming the product of 19 and 12 in the same moduli:

$$
\begin{aligned}
m_{i} & =(5,7,9,2) \\
\hline 19 & =(4,5,1,1) \\
12 & =(2,5,3,0) \\
\hline 19 \times 12 & =(3,4,3,0)=228,
\end{aligned}
$$

where 1 times 0 is 0 in any modulo, 3 times 1 is 3 modulo 9,5 times 5 is 25 , but modulo 7 the remainder or residue is 4 , and 4 times 2 is 8 , which yields a residue of 3 in modulo 5. The product of 19 and 12 in decimal is 228 . But $228 / 5$ equals 45 and a residue of 3 etc. from which $228=(3,4,3,0)$ with respective moduli $(5,7,9,2)$. From the above examples, the attractive features of residue arithmetic noted earlier are apparent.

\section{Decimal-to-Residue Conversion}

For the reasons indicated in Sec. II, considerable interest exists in residue arithmetic, especially optical residue arithmetic systems. In this section and the next one, we describe two specific coherent optical correlator architectures that perform decimal/residue/decimal conversion. These specific system architectures are presented to clarify the pulse-position coding, carrier modulation, and aperture control operations involved. The discussion applies directly to a general correlator. However, we defer presentation of this more general analysis to Sec. V after the above operations have been described for these specific system architectures in Secs. III and IV.

We consider the conversion of a decimal number into the residue number $R_{m_{i}}$ modulo $m_{i}$ and the specific correlator topology in Fig. 1. As noted earlier, we represent the input decimal number $X$ in pulse position code as the location of a spot of light in the input plane $P_{0}$. We define a unit decimal distance $\Delta x$ and thus represent the decimal number $x$ by a delta function at $x_{0}=X \Delta x$ in $P_{0}$. The amplitude transmittance of $P_{0}$ is thus

$$
g\left(x_{0}\right)=\delta\left(x_{0}-X \Delta x\right) .
$$

Lenses $L_{1}$ in Fig. 1 form the 1-D Fourier transform (FT) of g horizontally, while imaging vertically (i.e., a multichannel 1-D FT). We consider only one $y$ channel for simplicity. The FT of $g$ incident on $P_{1}$ is 


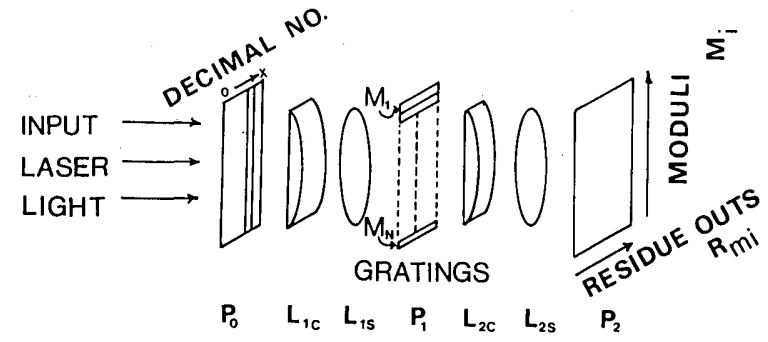

Fig. 1. Schematic diagram of a decimal-to-residue optical converter.

$$
G(u)=\exp (j 2 \pi u X \Delta x) .
$$

Spatial distance $x_{1}$ in $P_{1}$ is related to input spatial frequency $u$ by

$$
x_{1}=\lambda f_{L} u,
$$

where $\lambda$ is the wavelength of the laser light used, and $f_{L}$ $=f_{L S 1}$ is the focal length of lens $L_{1 s}$ in Fig. 1 . Equation (2) describes a planewave incident on $P_{1}$ at an angle that depends on $X \Delta x$ (the position of the input delta function or hence the value of the decimal input number $X)$.

For simplicity we consider only one vertical channel of the multichannel system and the conversion of $X$ to $R_{m_{i}}$ modulo $m_{i}$. At $P_{1}$ in Fig. 1, we place a square wave grating with transmittance

$$
H_{i}(u)=\sum_{n=-\infty}^{\infty} \exp \left(j 2 \pi u n m_{i} \Delta x\right),
$$

where the fundamental frequency $u_{g i}$ of the grating present on this channel $i$ is chosen such that the separation between the dc and \pm first order terms in the FT of Eq. (4) is

$$
m_{i} \Delta x=u_{g i} \lambda f_{L} .
$$

For simplicity, the same unit distance $\Delta x$ is assumed for decimal units in $P_{0}$ and for residue units in $P_{2}$. (This is equivalent to assuming a 1:1 imaging system for $L_{1}$ and $L_{2}$.)

In one version of a decimal/residue converter, the input $P_{0}$ datum is a vertical slit (one decimal number), and the desired outputs at $P_{2}$ are the residue numbers $R_{m_{i}}$ for $N$ moduli $m_{i}$ as shown in Fig. 1 . As we discuss in Secs. VII and VIII, this is not the only or optimum formulation of decimal/residue conversion when over-all system integration is considered. However, for our present purposes the above scenario of converting $X$ to the $N$ residue $R_{m_{i}}$, where there are $N$ moduli $m_{i}$ is adequate to convey the important system features. In such a case, the system will have $N$ channels, and at $P_{1}$ there will be $N$ different square waves recorded on these $N$ channels. The frequency $u_{g i}$ of the grating on channel $i$ will correspond to the modulus $m_{i}$ of that channel as in Eq. (5). Again, for simplicity, we restrict attention at present to one channel $i$.

The light amplitude distribution leaving channel $i$ of plane $P_{1}$ is the product of Eqs. (2) and (4) or

$$
G H_{i}=\sum_{n=-\infty}^{\infty} \exp \left[j 2 \pi u\left(X-n m_{i}\right) \Delta x\right] .
$$

Lenses $L_{2}$ in Fig. 1 form the 1-D horizontal FT of Eq. (6), and at $P_{2}$ we find

$$
f_{2}\left(x_{2}\right)=\sum_{n=-\infty}^{\infty} \delta\left[x_{2}-\left(X-n m_{i}\right) \Delta x\right],
$$

where $x_{2}$ is the spatial coordinate of $P_{2}$. This $P_{2}$ pattern consists of delta functions with center-to-center spacings $m_{i} \Delta x$ proportional to the modulus $m_{i}$ and replicated at distances that are multiples of this modulus. These delta function outputs occur at

$$
x_{2}=\left(X-n m_{i}\right) \Delta x .
$$

The pulse position coding of $X$ in $P_{0}$ and the carrier modulation [multiplication of Eq. (2) by Eq. (4) and Fourier transforming the result] operations involved have now been explained. Before describing the last required operation (aperture control), we digress for the moment to a general description of decimal/residue conversion. Any decimal number $X$ can be written as

$$
X=n m_{i}+R_{m_{i}},
$$

where $n$ is the number of times $X$ is divisible by $m_{i}$ and where the remainder or residue $R_{m_{i}}$ must satisfy

$$
0 \leq R_{m_{i}} \leq m_{i}-1 \text {. }
$$

Solving Eq. (9) for $R_{m_{i}}$, we find

$$
R_{m_{i}}=X-n m_{i} \text {. }
$$

From Eq. (11a), we see that $R$ is the remainder after $m_{i}$ has been subtracted $n$ times from $X$. Multiplying Eqs. (10a) and (11a) by $\Delta x$, we obtain

$$
\begin{aligned}
0 & \leq R_{m_{i}} \Delta x \leq\left(m_{i}-1\right) \Delta x, \\
R_{m_{i}} \Delta x & =\left(X-n m_{i}\right) \Delta x .
\end{aligned}
$$

Now, returning to Eq. (7), we see that the locations of the $n$ output delta functions in $P_{2}$ of Fig. 1 satisfy Eq. (8), which agrees with Eq. (11b). To insure that the subtraction of $m_{i}$ from $X$ has been performed the proper number of times $n$ so that Eqs. (10a) and (10b) are satisfied, we examine only the $P_{2}$ region satisfying

$$
0 \leq x_{2} \leq\left(m_{i}-1\right) \Delta x .
$$

This is obviously equivalent to satisfying Eqs. (10a) and (10b). Only one delta function output in Eq. (8) satisfies (12).

If we place a rectangular aperture at $P_{2}$ of Fig. 1 of width $m_{i} \Delta x$ in $x_{2}$ centered at $P_{2}$ as defined by Eq. (12), there will be $m_{i}$ possible locations of the output spot of light within this aperture. The location of the output peak of light within this aperture denotes the residue $R_{m_{i}}$ modulo $m_{i}$ of the decimal input $X$ in terms of a unit residue distance $\Delta x$ (i.e., pulse-position coded). By aperture control we simply mean placing an aperture at $P_{2}$ to select the remainder or residue in the proper range. An experimental demonstration of decimalto-residue conversion is provided in Sec. VII. 
The above analysis considered only one channel of the system of Fig. 1. If this system is to be used to convert the decimal number $X$ into the $N$ residues $R_{m_{i}}$ for the $N$ moduli $m_{i}$, the input $P_{0}$ pattern is a slit as shown in Fig. 1. Its $x_{0}$ location $X \Delta x$ denotes the decimal input number $X$. At $P_{1}$, we now place $N$ gratings of spatial frequencies $u_{g i}$ (corresponding to the $N$ moduli $m_{i}$ to be used to represent $X$ ) on $N$ separate lines or channels at $P_{1}$. Within the aperture placed at $P_{2}$ there will be $N$ channels with an output spot of light at a different horizontal location on each channel. The horizontal location of the output on each channel $i$ denotes the residue number $R_{m_{i}}$ for $X$ in the modulus $m_{i}$ for the particular channel. As implied earlier, this particular arrangement of a decimal-to-residue converter may not be optimum from a systems standpoint. This issue and another decimal-to-residue converter scenario are described in Secs. VII and VIII.

\section{Residue/Decimal Conversion}

All operations within a residue arithmetic computer are of course performed in residue number notation. However, the input and output data for such a processor should be in conventional decimal (or binary etc.) form. The decimal-to-residue optical converter described in Sec. III addressed the input conversion required and introduced the pulse-position coding, carrier modulation, and aperture control concepts employed. In this section, we consider the conversion from residue-todecimal performed at the processor's output.

The schematic of a residue-to-decimal optical converter is shown in Fig. 2. The system is topologically the same as that of Fig. 1. However, the inputs at $P_{0}$ are now $N$ residue numbers in the $N$ moduli $m_{i}$ chosen. These $N$ input residue numbers $R_{m_{i}}$ are represented by a pulse of light whose horizontal distance $R \Delta x$ along channel $n$ represents the residue number by pulseposition coding as before.

At $P_{1}$ of Fig. 2, $N$ gratings are placed on the $N$ different channels with the spatial frequency of each grating chosen to correspond to the modulus $m_{i}$ for that channel. The output along a given channel $n$ at $P_{2}$ of Fig. 2 will be a set of delta functions as before. The horizontal positions of these delta functions now correspond to all possible decimal numbers to which the input residue number could correspond (for the particular modulus of that channel). The horizontal position $X \Delta x$ in $P_{2}$ at which the delta functions on all $N$ channels are aligned denotes the proper decimal output $X$ corresponding to the $N$ input residue numbers $R_{m_{i}}$ in the $N$ moduli $m_{i}$. To detect the output, a set of horizontally oriented linear photodiode or CCD selfscanned detector arrays can be placed at $P_{2}$. The output from an $N$ input NOR or AND gate (depending on the polarity outputs desired) in synchronization with a $\Delta x$ counter will denote the decimal output value $X$ (or equivalently the horizontal $X \Delta x$ position in $P_{2}$ at which all delta functions on all channels are present).

The optical realization suggested in Fig. 2 is a spatially multiplexed on-line version of a scheme suggested earlier by Huang. ${ }^{5}$ In the version in Ref. 5 (Fig. 6),

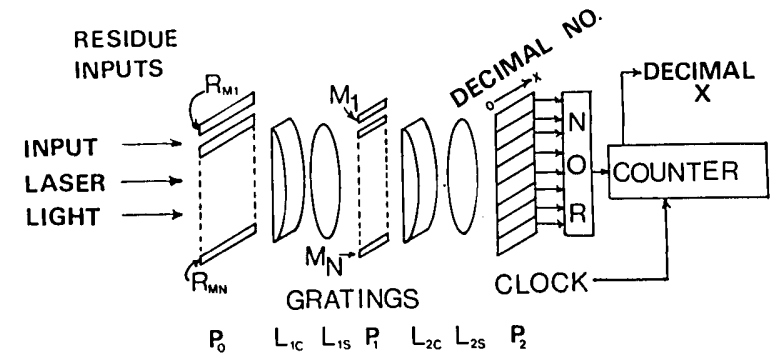

Fig. 2. Schematic diagram of a residue-to-decimal optical converter.

input light was passed sequentially through gratings (corresponding to each $m_{i}$ ). However, the lateral positions of the gratings had to be adjusted to correspond to the $R_{m_{i}}$ inputs to achieve the proper superposition at the output. In our scheme suggested in Fig. 2, mechanical motion of the gratings is not required.

\section{v. Correlation Formulation}

The residue arithmetic systems described in Secs. III and IV are linear and space invariant and can thus be described in correlation terminology. The following general correlation formulation of residue operations will be more clear now that detailed descriptions of the two converters in Secs. III and IV have been provided. How the designs in Figs. 1 and 2 originated is clear from the following formulation. In fact, an attractive feature of this correlation-based description is that the system architecture follows directly from the analytical formulation. As stated at the onset (Sec. I), a correlation formulation is most general since it allows realization of residue operations using other technologies. We have selected a coherent optical realization because it is one of the easiest to demonstrate and because of the real-time and parallel-processing advantages of such processors.

We consider the decimal-to-residue conversion of $X$ to $R_{m_{i}}$ modulo $m_{i}$. Now that the pulse-position coding, carrier modulation, and aperture control concepts have been described in Sec. III, the general description of the system as a correlator is straightforward. The input is a decimal number $X$ represented by $g\left(x_{0}\right)=\delta\left(x_{0}-\right.$ $X \Delta x)$. The desired residue output $R_{m_{i}}$ is $f\left(x_{2}\right)=\delta\left[x_{2}\right.$ $\left.-\left(X-n m_{i}\right) \Delta x\right]$. The required system impulse response needed to convert $g$ into $f$ is

$$
h\left(x_{0}\right)=\sum_{n} \delta\left(x_{0}-n m_{i} \Delta x\right),
$$

where $h$ described by Eq. (13) is simply the FT of the grating at $P_{1}$ of Fig. 1 described by Eq. (4).

Since the system's impulse response is real and symmetric, the convolution and correlation operations are equivalent, and the system's output at $P_{2}$ is the correlation of $g$ and $h$ :

$$
\begin{aligned}
g \circledast h & =\sum_{n} \int \delta\left(x_{0}-X \Delta x\right) \delta\left(x_{0}-n m_{i} \Delta x+x_{2}\right) d x_{0} \\
& =\sum_{n} \int \delta\left(x_{0}+x_{2}\right) \delta\left[x_{0}-\left(X-n m_{i}\right) \Delta x\right] d x_{0} \\
& =\sum_{n} \delta\left[x_{2}-\left(X-n m_{i}\right) \Delta x\right] .
\end{aligned}
$$




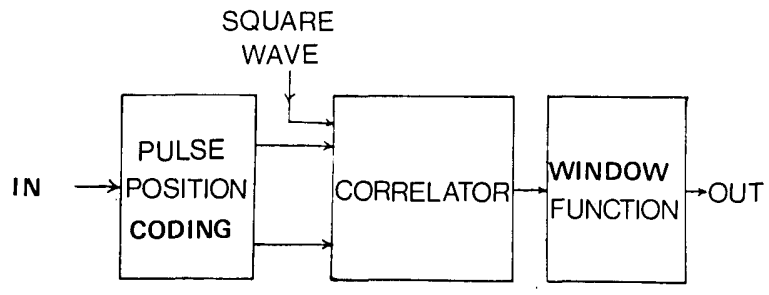

Fig. 3. General correlator schematic for a decimal/residue converter.

As seen, Eq. (14) is the desired output. The minimum positive location $x_{2}$ of the output delta function in the $P_{2}$ interval $0 \leq x_{2} \leq\left(m_{\mathrm{i}}-1\right) \Delta x$ (where $x_{2}$ is now the shift parameter in the correlation or equivalently the output coordinate) is the desired residue output $R_{m_{i}}$. Residue-to-decimal conversion can be formulated similarly.

The general system block diagram for a residue converter is shown in Fig. 3. The input is pulse-position coded by a deflector or other component, the correlator can be realized using many technologies, the output window function is equivalent to looking only in the proper region of output correlation space.

\section{Residue Arithmetic Optical Adder}

To demonstrate the utility of the correlation formulation of residue arithmetic, we describe the addition of the two residue numbers $R_{a}$ and $R_{b}$ modulo $m_{i}$ as a correlation. We then sketch the design of a residue arithmetic adder based on this formulation.

The two numbers to be added are represented in pulse-position coding by

$$
\begin{aligned}
& a=\delta\left(x_{0}-R_{a} \Delta x\right) \\
& b=\delta\left(x_{0}-R_{b} \Delta x\right) .
\end{aligned}
$$

The desired sum is

$$
\left|R_{a}+R_{b}\right|_{m_{i}}
$$

where the subscript $m_{i}$ denotes that the output of the sum is the residue modulo $m_{i}$. To produce the desired result, we form the correlation of $a$ and the mirror image of $b$,

$$
\begin{aligned}
a \circledast b & =\int_{-\infty}^{\infty} \delta\left(x-R_{a} \Delta x\right) \delta\left(x+R_{b} \Delta x+x^{\prime}\right) d x \\
& =\int_{-\infty}^{\infty} \delta\left[x-\left(R_{a}+R_{b}\right) \Delta x\right] \delta\left(x+x^{\prime}\right) d x \\
& =\delta\left[x^{\prime}-\left(R_{a}+R_{b}\right) \Delta x\right] .
\end{aligned}
$$

From Eq. (17), we see that the output of the above correlation is a delta function located in correlation space at $x^{\prime}=\left(R_{a}+R_{b}\right) \Delta x$, where $x^{\prime}$ is the correlation shift parameter. This corresponds to the desired sum of the two residue numbers pulse-position coded. To convert this sum to the residue modulo $m_{i}$, we use a grating and output aperture as in Fig. 1 to produce the desired result in Eq. (16).
The schematic of an optical system to realize these operations is shown in Fig. 4. The two pulse-position coded input residue numbers to be added are placed side by side in the input plane $P_{0}$ of a joint transform correlator. We describe the transmittance of $P_{0}$ in more conventional optical notation (in 1-D for the case of one modulus only for simplicity) by

$$
t_{0}\left(x_{0}\right)=\delta\left(x_{0}-x_{a}\right)+\delta\left(x_{0}+x_{b}\right),
$$

where $x_{a}=R_{a} \Delta x$ and $x_{b}=R_{b} \Delta x$ denote the locations of the two delta functions (for $R_{a}$ and $R_{b}$ ) in $P_{0}$. Lens $L_{1}$ forms the 1-D Fourier transform at $P_{1}$ of $t_{0}$ in the $x$ direction while imaging in the $y$ direction.

The light distribution in 1-D incident on $P_{1}$ is thus

$$
f_{1}(u)=\exp \left(j 2 \pi u x_{a}\right)+\exp \left(-j 2 \pi u x_{b}\right) .
$$

An optically addressed spatial light modulator ${ }^{10}$ (SLM) is placed at $P_{1}$. The transmittance of $P_{1}$ after exposure is $\left|f_{1}\right|^{2}$ or

$$
\begin{aligned}
t_{1} & =\left|\exp \left(j 2 \pi u x_{a}\right)+\exp \left(-j 2 \pi u x_{b}\right)\right|^{2} \\
& =2+2 \cos \left[2 \pi u\left(x_{a}+x_{b}\right)\right] .
\end{aligned}
$$

This SLM is read in reflection from the beam splitter BS in Fig. 4. Lens $L_{2}$ in Fig. 4 images $P_{1}$ onto $P_{2}$. At $P_{2}$, we place a grating whose transmittance in 1-D for the $y$ channel of concern we represent by

$$
t_{2}=\sum_{n} \exp \left(-j 2 \pi u n m_{i} \Delta x\right) .
$$

The light distribution leaving $P_{2}$ is now

$$
t_{1} t_{2}=\sum_{n} \exp \left[j 2 \pi u\left(x_{a}+x_{b}-n m_{i} \Delta x\right)\right] .
$$

Lens $L_{3}$ in Fig. 4 forms the FT of Eq. (22) at $P_{3}$. This $P_{3}$ pattern is a set of delta functions, as in Sec. III,

$$
\begin{aligned}
f_{3}=\sum_{n} \delta\left[x_{3}-\left(x_{a}+x_{b}-n m_{i} \Delta x\right)\right] & \\
& =\sum_{n} \delta\left[x_{3}-\left(R_{a}+R_{b}-n m_{i}\right) \Delta x\right] .
\end{aligned}
$$

The location of the delta function at $P_{3}$ of Fig. 4 in the range $0 \leq x_{3} \leq\left(m_{i}-1\right) \Delta x$ provides the desired residue of the sum of the two residue numbers modulo $m_{i}$.

In practice $a$ and $b$ are represented by $N$ residue numbers (for the case of $N$ moduli $m_{i}$ ). One way in which the contents of $P_{0}$ in Fig. 4 can be arranged to add two residue numbers with $N$ moduli is to allot $N$ channels in each half of plane $P_{0}$. The $N$ pairs of residue

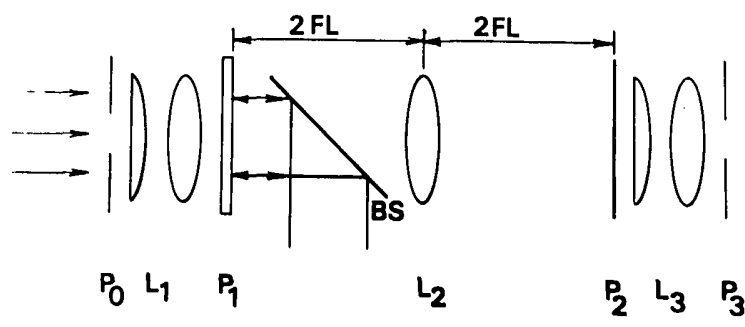

Fig. 4. Schematic diagram of a residue arithmetic optical adder. 
numbers $R_{a m_{i}}$ and $R_{b m_{i}}$ for the $N$ moduli can be recorded on these $N$ separate channels in pulse-position code. For this input data arrangement, we would use $N$ gratings at $N$ different spatial frequencies (corresponding to the $N$ moduli) on $N$ lines at $P_{2}$ of Fig. 4. At $P_{3}$ of Fig. 4, there will also be $N$ channels with the position of the delta function on each vertical channel proportional to the residue of the sum of the corresponding residue numbers in the corresponding modulus of that channel. The adder element shown in Fig. 4 is one basic elemental building block of a residue arithmetic system. (A multiplier can be realized by successive additions.)

\section{Experimental Demonstration}

The system of Fig. 1 was assembled to demonstrate and verify the decimal/residue conversion method described in Secs. III and V. To keep the unit decimal distance $\Delta x_{0}$ in $P_{0}$ and the unit residue distance $\Delta x_{2}$ in $P_{2}$ equal to $\Delta x$, a $1: 1$ imaging system was used. The focal lengths of both cylindrical lenses were $f_{L c}=$ 300 -mm with $f_{L s}=762$-mm focal lengths for both spherical lenses.

As noted several times earlier, the optimum deci$\mathrm{mal} / \mathrm{residue}$ converter need not always convert a decimal input number into its $N$ residue numbers. To demonstrate an alternate scenario and to still demonstrate the basic principle of decimal/residue conversion as a correlation, the 21 decimal numbers 0 to 20 were used as the inputs on 21 channels in $P_{0}$ of Fig. 1 . We consider the conversion of these 21 decimal inputs to 21 residue numbers all in the same modulus. The input used [Fig. 5(a)] consists of an impulse on each of the 21 vertical channels in $P_{0}$. The horizontal position of each of these impulses denotes a different decimal input number. Since the spacings between impulses on successive channels are equal to $\Delta x=0.7 \mathrm{~mm}$ (the unit input decimal distance), the decimal input on the bottom channel 0 is 0 , the number on channel 1 is 1 , etc., up to the top channel, which represents the decimal number 20 in pulse-position code.

This input pattern was produced by superimposing a tilted slit over a 1.4 -cycle/mm grating. The input pulses are then separated by $1 / 1.4 \simeq 0.7 \mathrm{~mm}=\Delta x$. Since the duty cycle of the 10 -cycle/mm grating was only $6 \%$, both odd and even harmonics of the fundamental grating frequency are present. The width of the input pulse is only $0.06(0.7) \mathrm{mm} \simeq 40 \mu \mathrm{m}$ or far less than the $0.7-\mathrm{mm}$ decimal limit used. The capacity of the system is thus not even approached by the example shown.

At $P_{1}$ of Fig. 1, we place a grating of spatial frequency $u_{g}=10$ cycles $/ \mathrm{mm}$. From Eq. (5), we see that $\Delta x$ and $u_{g}$ must satisfy

$$
\Delta x=0.7 \mathrm{~mm}=u_{g} \lambda f_{L} / m_{i}
$$

from which the modulus corresponding to the grating frequency used is found to be $m_{i}=7$. Thus the system of $\mathrm{Fig}$. 1, as designed above, will convert the 21 pulseposition coded decimal numbers [Fig. 5(a)] into residue numbers modulo $m_{i}=7$.
The next step is to position properly the output plane $P_{2}$ aperture. To do this, we must calibrate the system. Since the residues modulo 7 of the decimal numbers 0 , 7 , and 14 are all 0 , we chose input channel 7 for calibration purposes. With only the input impulse on channel 7 present, the $P_{2}$ pattern in Fig. 1 consists of a dc, \pm 1 order, etc. spots of light. We position a rectangular aperture of width $7 \Delta x$ in $P_{2}$ such that the dc spot appears just inside the left edge of the aperture (when viewed from the detector plane). The -1 order spot is cut off and lies just outside the edge of this aperture. The system is now aligned.

With the full input plane $P_{0}$ pattern [Fig. 5(a)] illuminated, the output plane $P_{2}$ pattern of Fig. 5(b) results. It contains 21 vertical channels (corresponding to the decimal numbers 0 to 20 on the 21 input channels). On each vertical channel, the horizontal position of the output spot (within the rectangular aperture at $P_{2}$ ) lies in one of seven possible locations. These locations correspond to the seven possible residue numbers 0 to 6 .

The residues modulo 7 of the decimal numbers 0 to 6 are just 0 to 6 , respectively. In these cases the dc spot simply shifts across the $P_{2}$ aperture from left to right.
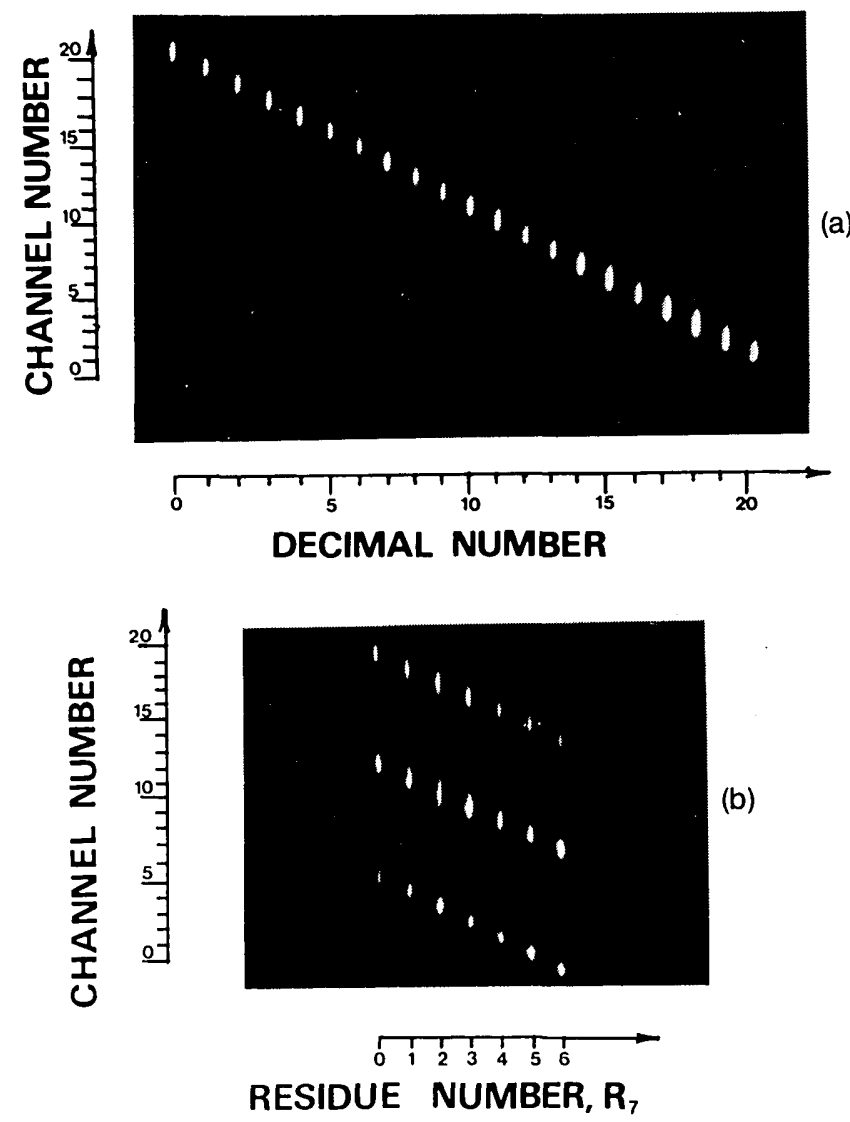

Fig. 5. Experimental demonstration of the conversion of the 21 decimal input numbers 0 to 20 into residue numbers modulo 7 using the optical system in Fig. 1: (a) input $P_{0}$ pattern; (b) output $P_{2}$ pattern. 
For the decimal input 7, the dc spot now shifts out the right-hand side of the $P_{2}$ aperture, and the first-order spot enters the left-hand side of the $P_{2}$ aperture. Thus the correct residue number 0 results. Use of a high threshold SLM (or a thresholded TV monitor) at $P_{2}$ can restore the intensity of all spots of light to the same level.

To demonstrate residue-to-decimal conversion, the system of Fig. 2 was assembled with the same lenses used in the above experiment. The conversion performed was of the residue number $(1,1,3)$ with moduli $(3,4,5)$ into its decimal equivalent 13 . Only three input channels are required for this case. The unit distance $\Delta x$ for $P_{0}$ and $P_{2}$ of Fig. 2 was now $0.48 \mathrm{~mm}$. The three gratings used on the three channels at $P_{1}$ were at 3,4 , and 5 cycles $/ \mathrm{mm}$ (corresponding to the three moduli $m_{1}$ $=3, m_{2}=4$, and $m_{3}=5$ ).

The three input residue numbers were pulse-position coded on three channels at $P_{0}$ of Fig. 2 with $\Delta x=0.48$ $\mathrm{mm}$ as shown in Fig. 6(a). The resultant output plane $P_{2}$ pattern in Fig. 2 is shown in Fig. 6(b). The horizontal locations of the impulses on each output channel correspond to all the possible decimal numbers that could be represented by the corresponding residue input in the specific modulus chosen. As seen in Fig. 6(b), impulses from all three channels occur only at the horizontal location 13. Thus the decimal equivalent of the residue input $(1,1,3)$ is found to be 13 , and residue-todecimal conversion has been demonstrated.

\section{Discussion}

The dynamic range of an optical residue arithmetic processor is the product of the moduli used, this is also the largest number $M$ that can be represented in residue. As the number of moduli increases, each required subcalculation (recall that all calculations in all moduli are performed in parallel) needs only a dynamic range equal to the modulus chosen. In the pulse-position coding scheme used, $M$ resolution elements are needed in the input plane $P_{0}$ of Fig. 1 and the output plane $P_{2}$ of Fig. 2 to represent the full range of decimal numbers possible. Thus the system's most severe bandwidth and dynamic range requirements occur during the input and output conversions, since the full dynamic range of the decimal number must be realized in these stages.

The resolution $\Delta x$ possible and needed in $P_{0}$ of Fig. 1 is related to $M$ and the input aperture $A$ by

$$
M=A / \Delta x .
$$

To increase $M$, we must increase $A$ and decrease $\Delta x$. The upper limit on $A$ is set by the lens system following $P_{0}$. The lower limit for $\Delta x$ is determined by the minimum resolvable spot size and geometrical accuracy with which the input can be recorded. The lens sets $L_{1}$ and $L_{2}$ in Fig. 1 must be capable of producing Fourier spots whose position is linearly related to spatial frequencies in the input plane of the lens. We investigated the positional accuracy of an FT lens ${ }^{10}$ and found it to be better than $0.06 \%$. In general the accuracy and fidelity with which input data can be recorded limit the performance of an optical processor. Thus, we expect $\Delta x$ and positional inaccuracies in recording the input data to limit the dynamic range of an optical residue arithmetic system.

The motivation for considering an optical implementation of a residue arithmetic processor was to increase the dynamic range and accuracy obtainable in an optical system. However, the approximately 1000 point linear SBWP presently obtainable with scanners and spatial light modulators ${ }^{11}$ still does not permit more than a 10-bit dynamic range (if the pulse-position coding scheme and optical systems described earlier are used). Two approaches by which increased input space-bandwidth and hence system dynamic range can be obtained are now discussed.

The first is the use of input numbers in binary rather than decimal notation. For an input dynamic range of 64,000 we require 16 bits to represent the input data in binary notation (compared with a linear input SBWP of 64,000 for the pulse-position coded decimal representation of the largest input number). However, a binary/residue converter is now required. Such an optical system can be realized using the joint transform correlator adder of Fig. 4 for $B$ (equal to the number of
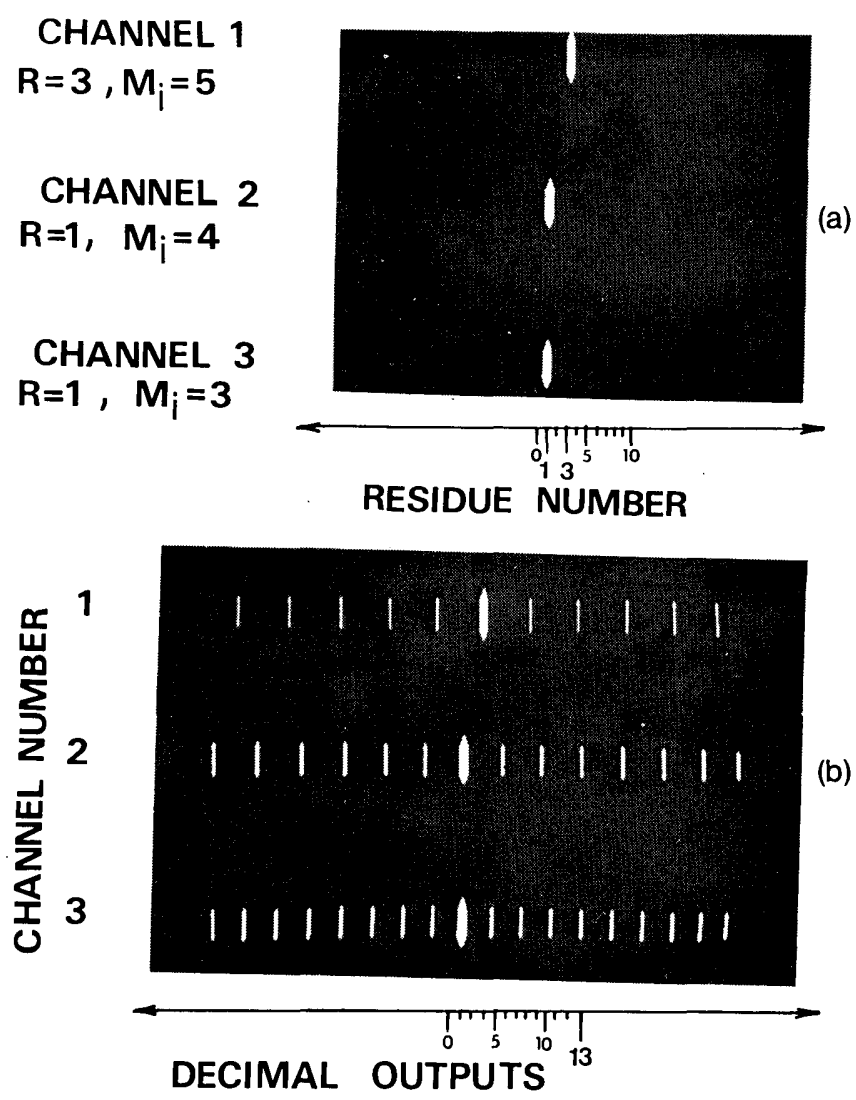

Fig. 6. Experimental demonstration of the conversion of the residue number $(1,1,3)$ with moduli $(3,4,5)$ into the decimal number 13 using the optical system of Fig. 2: (a) input $P_{0}$ pattern; (b) output $P_{2}$ pattern. 


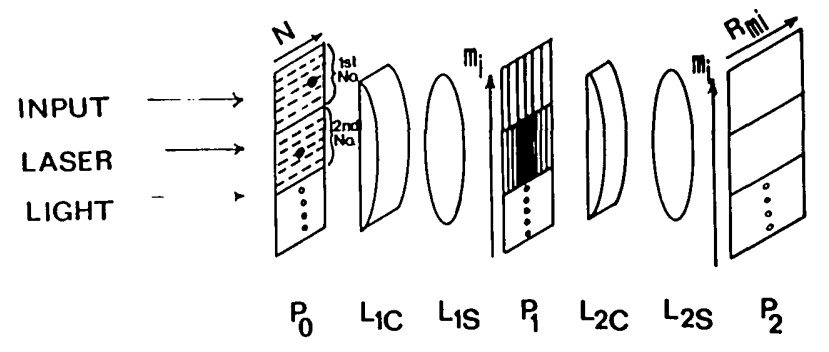

Fig. 7. Schematic diagram of a high dynamic range decimal to residue converter using raster recorded input data.

bits) cycles. To visualize such a system, recall that $N$ in decimal notation is related to its binary representation $\left(a_{0}, a_{1}, a_{2} \ldots a_{B}\right)$ by

$$
N=\sum_{n=0}^{B} a_{b} 2^{b},
$$

where each $a_{b}$ is 0 or 1 . In residue notation $N$ modulo $m_{i}$ is

$$
|N|_{m_{i}}=\left(a_{0}\left|2^{0}\right|_{m_{i}}+a_{1}\left|2^{1}\right| m_{i}+a_{2}\left|2^{2}\right|_{m_{i}}+\ldots\right)_{m_{i}} .
$$

From Eq. (26), we realize that $N$ can be represented modulo $m_{i}$ as the residue sum of $B$ numbers $2^{b}$ in residue, and that these $2^{b}$ modulo $m_{i}$ values are known in advance. In essence, we can realize Eq. (26) by adding 0 or $2^{b}$ to a running residue sum depending on whether the corresponding binary bit of $N$ is 0 or 1 . The resultant output after the addition of the most significant bit is then $|N|_{m_{i}}$ in residue modulo $m_{i}$. Realization of this conversion by the cyclic operation of one adder of the type shown in Fig. 4 is preferable to a cascade of $B-1$ such adders.

However, an alternate method exists whereby the dynamic range of $N$ can be increased, without exceeding the assumed input linear SBWP of 1000 . This approach involves raster recording the input data with zero retrace time and use of the folded spectrum optical spectrum analyzer. ${ }^{2}$ In the optical residue arithmetic version of such a high dynamic range raster recorded decimal/residue converter, the pulse position coding of $N$ is still employed. However, $K$ lines at $P_{0}$ of Fig. 1 are used to represent $N$ as

$$
N x_{0}-K N_{L} x_{0}=\left(N-K N_{L}\right) x_{0} .
$$

In other words, for a maximum $N=10,000$ and a linear positional or resolutional $N_{L}=1000$, we use $K=10$ lines at $P_{0}$ to represent $N$. If $0 \leq N \leq 1000$, a delta function of light appears on line $K=1$. If $1000 \leq N \leq$ 2000 , the delta function appears on line $K=2$ at $P_{0}$ etc. If we use $N=n m_{i}+R_{m_{i}}$ and assume $K N_{L}=r m_{i}+$ $R_{m i}^{\prime}$,

$$
N-K N_{L}=(n-r) m_{i}+R_{m_{i}}-R_{m i}^{\prime},
$$

which reduces to

$$
R_{m_{i}}=\left(N-K N_{L}\right)-(n-r) m_{i}
$$

when $N_{L}$ is a multiple of $m_{i}$, since then $R_{m_{i}}^{\prime}=0$.
As a numerical example, consider the conversion of $N=168$ to residue modulo 5 assuming a maximum $N_{L}$ $=50$. In our raster recorded version of the input $P_{0}$ pattern in Fig. 1, the delta function corresponding to $N$ $=168$ occurs 18 spaces $(18 \Delta x)$ from the left edge of the $k=4$ line in $P_{0}$. Since $N_{L}=50$ is an integer multiple of $m_{i}=5, N=0-49$ appear on line $k=1, N=49-99$ appear on line 2 , etc. Then,

$$
18_{5}=(168-150)_{5}=168_{5}-150_{5}=168_{5} .
$$

The schematic diagram for such a system is shown in Fig. 7. With $K$ lines required at $P_{0}$ to represent $N$, the $m_{i}$ grating at $P_{1}$ is also replicated for $K$ lines (assuming 1:1 imaging optics for $\left.L_{1}\right)$. A second grating at a spatial frequency corresponding to the second $m_{i}$ is recorded on the next $K$ lines at $P_{1}$ (or alternatively a grating at one $u_{g i}$ is present at $P_{1}$ depending upon the system scenario). In all cases only one spot appears within the first $K$ lines of the $P_{2}$ aperture etc., and thus as before the horizontal locations of the peaks of light at $P_{2}$ correspond to the desired $R_{m i}$. The vertical $P_{2}$ axis is now partitioned into sets of $K$ lines with each set corresponding to one $R_{m i}$. Since the 2-D SBWP of available spatial light modulators ${ }^{11}$ is so large, this utilization of available real estate at $P_{0}$ is warranted if the desired system dynamic range is to be realized. One shortcoming of the system of Fig. 7 is the low usable input light level.

This can be improved if the decimal input number is used to control an oscillator to raster record the input as a signal at frequency $f_{N}$ proportional to $N$ on $K$ lines. The 2-D Fourier transform of such a raster-recorded input pattern contains coarse and fine frequency axes. The location of the output peak along the fine frequency axis is the desired residue modulo, the input horizontal line scan rate. ${ }^{12}$ The modulus is thus set by the input line scan rate, and a set of gratings is not required. ${ }^{13}$

At the output plane $P_{2}$ of Figs. 1 or 7 , another accuracy issue arises. Positional accuracy represents no major problem since only $m_{i}$ resolution points per channel are needed. Rather, the diffraction efficiency of the grating used at $P_{1}$ must be sufficient to produce enough detectable light in the $\mathrm{m} / \mathrm{m}_{i}$ th order of the grating. The problem can be relieved by use of larger $m_{i}$ values, by use of blazed or bleached gratings, etc. The accuracy of the spatial frequency of this grating will also affect performance, but this is not expected to be a major error source since the grating need be produced only once.

Since the aperture of the grating at $P_{1}$ determines the spot size in the output plane $P_{2}$, we must insure that this aperture remains above some minimum value for all beams emerging from different locations in $P_{0}$ that strike $P_{1}$ at different angles.

For simplicity in the analyses used, we assumed equal unit distances $\Delta x_{0}=\Delta x_{2}=\Delta x$ in $P_{0}$ and $P_{2}$. In Fig. 1, the $P_{0}$ resolution must be superb. However, there is no reason to retain fine resolution in $P_{2}$ since only $m_{i}$ points per line are needed in $P_{2}$. Thus, in practice choosing $m_{i}$ large and $\Delta x_{2}>\Delta x_{0}$ should improve per- 
formance. However, utilization of the available space bandwidth (SBW) and a full system design will determine the optimum values.

We demonstrate by example this latter point and why the particular input format for the converters of Figs. 1 and 2 and the adder of Fig. 4 may not be as indicated in the examples in Secs. III, IV, and VI. Consider the input SBW requirements for a residue adder to add in parallel 100 pairs of numbers, each of which is described in residue by $N=11$ moduli (with the largest modulus equal to 31 ). Since $\max \left(m_{i}\right)=31$ and $N=11$, we require a SBW $=343$ to represent fully one number in residue. To represent 100 numbers, we require a SBW $=34,300$. Thus to add two such pairs of 100 decimal numbers, we require an input $\mathrm{SBW}=68,600$.

This value may seem quite formidable, and in fact it is for many systems. However, the input SBW achievable in real time on a spatial light modulator ${ }^{11}$ in a coherent optical processor easily exceeds $10^{3} \times 10^{3}=$ $10^{6}$. Thus the input SBW required for the above example leaves 931,400 of the $10^{6}$ possible input pixels unused. As this numerical example rather vividly demonstrates, the increased SBW requirements of a residue arithmetic system represent no problem in an optical residue arithmetic system. Thus, utilization of the large available input SBW of an optical processor will definitely require far more elaborate input formats than those described in the examples in Secs. II-VII. Detailed formulation of these aspects is a system integration problem that is best addressed for a specific signal or image processing problem. For now, we simply note that for these reasons a general formulation of residue arithmetic operations in correlation terminology is preferable since available technologies can then be used to realize the required system without reliance on special purpose components such as cascades of integrated optical switches that are not yet available.

In the numerical example presented in which $N=11$ moduli with $\max \left(m_{i}\right)=31$, the largest input number that can be represented is about $2^{42}$. Thus, this system with eleven moduli has the equivalent accuracy and dynamic range of a 42-bit digital computer.

A final note on system accuracy is merited. There are at least three distinguishable methods by which residue numbers can be represented in an optical processor. These are: the state of polarization or phase of the light at a single point and by the position of a spot of light. We have chosen to use the latter pulse-position coding representation. This allows direct formulation of the required processing in correlation terms, which in turn allows us to obtain a quantitative measure of the system's accuracy. If polarization or phase coding were used, analysis of system errors is far more complex, and SLMs are needed with higher uniformity and optical quality than are presently available. Analysis of the effect produced by errors in such space-variant components poses a complex statistical problem. In pulse position coding, the geometrical registration accuracy of the components are the dominant factors affecting system performance. Modeling and analysis of such geometrical effects are far more conducive to producing a quantitative error budget for the over-all system. Since an error of one unit in a residue number can cause large overall errors in the system, the error source analysis of such systems is vital. At present pulse position coding appears to be the most promising coding scheme to use.

\section{Summary}

Residue arithmetic has been shown to possess many features that make an optical numerical processor using residue arithmetic attractive. With no carries present, all operations can be performed in parallel thus using the parallel processing features of an optical system. Since a given computation is divided into a number of subcalculations (in different moduli), the dynamic range and hence the accuracy required for each subcalculation are far less than those required for the over-all calculation. Thus, the accuracy and dynamic range limitations often associated with optical systems are greatly reduced by the use of optical residue arithmetic.

A new decimal/residue/decimal optical conversion scheme has been described and experimentally demonstrated. Pulse-position coding, carrier modulation, and aperture control are used to achieve conversions. These operations and residue addition have been formulated in linear system theory and correlation terms. This approach allows one to implement directly the resultant system using many different technologies. The analysis and design of a residue adder has demonstrated the ability of this correlation-based formulation.

The support of the National Science Foundation, Engineering Division (contract ENG 77-20038), and motivation provided by Rome Air Development Center for the work reported upon is gratefully acknowledged.

\section{References}

1. H. L. Garner, IRE Trans. Electron. Comput. EC-8, 140 (1959).

2. N. S. Szabo and R. I. Tanaka, Residue Arithmetic and Its Applications to Computer Technology (McGraw-Hill, New York, 1967).

3. D. E. Knuth, Seminumerical Algorithms, Vol. 2 (Addison-Wesley, Reading, Mass., 1969), pp. 248-256.

4. A. Huang, in Proceedings of the International Optical Computing Conference Washington, D.C., April 1975, X. X. Blank, Ed. (IEEE New York, 1975), pp. 14-18.

5. A. Huang, Proc. Electro-Optical Systems Design Conference, Anaheim, October 1977, pp. 208-212.

6. S. Collins, Proc. Soc. Photo-Opt. Instrum. Eng. 128, (1977).

7. A. Huang, Y. Tsunoda, and J. W. Goodman, Appl. Opt. 18, 15 Jan 1979.

8. A. Huang, Y. Tsunoda, and J. W. Goodman, Stanford Electronics Lab Rept. 6422-1 (March 1978).

9. D. Casasent and T. Luu, Proc. Electro-Optical Systems Design Conference, Anaheim, October 1977, pp. 208-212.

10. D. Casasent and T. Luu, Appl. Opt. 17, 1701 (1978).

11. D. Casasent, Proc. IEEE 65, 143 (1977).

12. C. Thomas, Appl. Opt. 5, 1782 (1966).

13. This alternate raster recording scheme was suggested to us by $L$. Weiner of Ampex Corp. 